\title{
3-D reconstructions of active stars
}

\author{
Heidi Korhonen ${ }^{1,2,3}$ \\ ${ }^{1}$ Niels Bohr Institute, University of Copenhagen, Juliane Maries Vej 30, DK-2100 Copenhagen, \\ Denmark \\ ${ }^{2}$ Centre for Star and Planet Formation, Natural History Museum of Denmark, University of \\ Copenhagen, Øster Voldgade 5-7, DK-1350, Copenhagen, Denmark \\ ${ }^{3}$ Finnish Centre for Astronomy with ESO (FINCA), University of Turku, Väisäläntie 20, \\ FI-21500 Piikkiö, Finland
}

\begin{abstract}
Stars are usually faint point sources and investigating their surfaces and interiors observationally is very demanding. Here I give a review on the state-of-the-art observing techniques and recent results on studying interiors and surface features of active stars.
\end{abstract}

Keywords. stars: activity, atmospheres, interiors, rotation, spots

\section{Introduction}

The only star we can study easily with any spatial resolution is the Sun, and now with the twin STEREO spacecraft we can do it even in 3-D. For other stars we are usually restricted to using indirect methods for getting information of their surface structures. Here a short review on methods for studying stellar interiors and surfaces and the main recent results are presented.

\section{Interiors}

Kepler has not only revolutionised the exoplanet research, but also stellar astrophysics. The short, one minute, cadence data is uniquely suited for studying stellar oscillations and thus the interiors of stars (see, e.g., Gilliland et al. 2010). Recent Kepler results, based on a sample of 500 solar-like stars, show that even though distribution of observed radii is similar to the one predicted by models of synthetic stellar populations in the Galaxy, the distribution of observed masses seems to deviate significantly from the predicted distribution (Chaplin et al. 2011a). This result raises interesting questions on the star formation rate and initial mass function. Studying the same sample of solar-like stars further reveals that the number of stars showing oscillations decreases significantly with increasing levels of activity (Chaplin et al. 2011b). This on the other hand implies that the magnetic fields can inhibit the amplitudes of solar-like oscillations.

\section{Surfaces}

The strength of the dynamo-created stellar magnetic fields and the frequency of the associated phenomena (e.g., starspots and flares) is strongly linked to the rotation of the star, with rapid rotation enhancing the field generation (e.g., Pallavicini et al. 1981). Many active stars, rapid rotation usually induced either by youth or binarity, show large starspots which are detectable even using photometric observations from small groundbased telescopes (e.g., Kron 1947). In some cases the monitoring has become almost nightly after the implementation of automatic photometric telescopes in the late 1980's (e.g., Rodonò \& Cutispoto 1992; Strassmeier et al. 1997). These observations give crucial 
information on the stellar activity cycles (e.g., Oláh et al. 2009) and long-term spot longitudes (e.g., Lehtinen et al. 2012), i.e., 2-D information where one dimension is longitude and the other time. With observations using multiple filters crude spot latitudes can be obtained (e.g., Roettenbacher et al. 2011), making the reconstruction 3-D (longitude, latitude and time). Similarly, the continuous, high precision, space-based light-curves allow for recovering latitudinal and longitudinal information in addition to the temporal variations.

For obtaining more detailed stellar surface structures Doppler imaging techniques have to be used (e.g., Vogt, Penrod \& Hatzes 1987), giving a 2-D snapshot of the surface temperature with a reasonable resolution (e.g., Korhonen et al. 2010). Combining many observations from different epochs adds the third dimension, namely time (e.g., Skelly et al. 2010; Hackman et al. 2012). Doppler images can also be used for studying stellar surface rotation and its dependence on latitude (for a recent review see Korhonen 2012). If spectral lines formed at different heights in the stellar atmosphere are used in the reconstruction, also vertical structures in starspots can be mapped (Berdyugina 2011), coming closer to what is possible in the solar case.

\section{Conclusions and outlook}

Obtaining multi-dimensional infromation on the active stars, which are just point sources, is challenging but not impossible. Observations give us detailed information, both temporal and spatial, on occurrence of starspots. In the future long baseline infrared/optical interferometry can offer a direct check on some of the results. Furthermore, while the interferometric imaging is restricted to the brightest and nearest stars, the method does not require large projected rotational velocities, like Doppler imaging does. This will open up new targets that have not been approachable earlier, significantly widening the parameter space for activity studies.

Acknowledgments H.K. acknowledges the support from European Commission's Marie Curie IEF Programme, and an IAU travel grant to participate the General Assembly.

\section{References}

Berdyugina S. V. 2011, in: J. R. Kuhn, et al. (eds), ASP Conf. Ser. 437, 219

Chaplin, W. J., Kjeldsen, H., Christensen-Dalsgaard, J., et al. 2011a, Science 332, 213

Chaplin, W. J., Bedding, T. R., Bonanno, A., et al. 2011b, ApJ (Letters) 732, L5

Gilliland, R. L., Brown, T. M., Christensen-Dalsgaard, J., et al. 2010, PASP 122, 131

Hackman, T., Mantere, M. J., Lindborg, J., et al. 2012, A\& A 538, A126

Kron, G. E. 1947, PASP 59, 261

Lehtinen, J., Jetsu, L., Hackman, T., Kajatkari, P., \& Henry, G. W. 2012, A 6 A 542, A38

Korhonen, H. 2012, in C. H. Mandrini, \& D. F. Webb (eds), Proceedings of IAU Symposium 286 (Cambridge University Press), p. 268

Korhonen, H., Wittkowski, M., Kővári, Zs, et al. 2010, A\&A 515, A14

Oláh, K., Kolláth, Z., Granzer, T., et al. 2009, A\& $A$ 501, 703

Rodonò, M. \& Cutispoto, G. 1992, A\&AS 95, 55

Roettenbacher, R. M., Harmon, R. O., Vutisalchavakul, N., \& Henry, G. W. 2011, AJ 141, 138

Skelly, M. B., Donati, J.-F., Bouvier, J., et al. 2010, MNRAS 403, 159

Strassmeier, K. G., Bartus, J., Cutispoto, G., \& Rodono, M. 1997, A $\& A S$ 125, 11

Vogt, S. S., Penrod, G., \& Donald, Hatzes, A. P. 1987, ApJ 321, 496 